\title{
Melatonin reverses type 2 diabetes-induced cognitive deficits via attenuation of oxidative/nitrosative stress and NF-kB-mediated neuroinflammation in rat hippocampus
}

\author{
Zhang Xuyan ${ }^{1,2}$, Ye Jing Ping ${ }^{3}$, Wang Zhongjing ${ }^{1,2}$, Ding Sheng ${ }^{1,2}$, Li Li ${ }^{1,2}$, Yang \\ Fan $^{1,2}$, Mao Hong ${ }^{1,2 \star}$ \\ ${ }^{1}$ Department of Endocrinology, ${ }^{2}$ Key Laboratory for Molecular Diagnosis of Hubei Province, The Central Hospital of Wuhan, \\ Tongji Medical College, Huazhong University of Science and Technology, Wuhan 430014, ${ }^{3}$ Department of Pediatrics, Renmin \\ Hospital of Wuhan University, Wuhan University, Wuhan, Hubei 430060, China
}

*For correspondence: Email: maohong8787@163.com; Tel/Fax: 0086-027-82211468

Sent for review: 26 June 2017

Revised accepted: 24 November 2017

\begin{abstract}
Purpose: To evaluate the protective effect of melatonin on diabetes-induced cognitive dysfunction. Methods: Rats were fed a high-fat diet + streptozotocin (HFD + STZ) for 15 weeks to induce type 2 diabetes (HFD + STZ group). At the end of the 15-week induction of diabetes, cognitive function in the diabetic rats was estimated using a Morris water maze and an object recognition task. Next, the diabetic rats were treated with melatonin $(10 \mathrm{mg} / \mathrm{kg}$, po) for 3 weeks. Thereafter, cognitive function was reevaluated in the melatonin-treated diabetic rats (melatonin group).

Results: There was a significant $(p<0.01)$ decrease in the serum glucose and insulin in melatonintreated diabetes type 2 rats compared with that of diabetes type 2 rats exposed to only HFD + STZ. Treatment with melatonin $(10 \mathrm{mg} / \mathrm{kg}$, po) for 3 weeks in diabetic type 2 rats also caused a significant increase $(p<0.01)$ in the time spent in the target quadrant and preference index in diabetic rats compared with the HFD + STZ group. There were significant decreases in reactive oxygen species (ROS), oxido-nitrosative stress markers, including thiobarbituric acid reactive substances (TBARS), nitrite, and depleted glutathione (GSH) level in the hippocampus of melatonin-treated group, compared with the HFD + STZ-treated group. Moreover, the melatonin-treated group showed significant inhibition of nuclear factor kappa-light-chain-enhancer of activated B cells (NF-KB) and reduction in the levels of proinflammatory cytokines.

Conclusion: The results demonstrate that melatonin prevents cognitive dysfunction in type 2 diabetic rats by attenuating oxido-nitrosative stress and NF-KB-mediated neuroinflammation. This effect suggests that melatonin may be useful for the management of cognitive dysfunction in patients suffering from diabetes.
\end{abstract}

Keywords: Cognitive dysfunction, Melatonin, Neuroinflammation, Nuclear factor kappa-light-chainenhancer of activated $B$ cells (NF-KB), Oxido-nitrosative stress, Type 2 diabetes

\begin{abstract}
This is an Open Access article that uses a funding model which does not charge readers or their institutions for access and distributed under the terms of the Creative Commons Attribution License (http://creativecommons.org/licenses/by/4.0) and the Budapest Open Access Initiative (http://www.budapestopenaccessinitiative.org/read), which permit unrestricted use, distribution, and reproduction in any medium, provided the original work is properly credited.
\end{abstract}

Tropical Journal of Pharmaceutical Research is indexed by Science Citation Index (SciSearch), Scopus, International Pharmaceutical Abstract, Chemical Abstracts, Embase, Index Copernicus, EBSCO, African Index Medicus, JournalSeek, Journal Citation Reports/Science Edition, Directory of Open Access Journals (DOAJ), African Journal Online, Bioline International, Open-J-Gate and Pharmacy Abstracts 


\section{INTRODUCTION}

Diabetes is a metabolic disorder that results from inadequate action and secretion of insulin [1]. Complications in several body systems, such as cranial and peripheral nerves, large vessels, eyes, kidney, and cardiovascular system, are associated with diabetes [2,3]. Cognitive deficits have also been observed in both type 1 and type 2 diabetes patients.

Previous studies suggest that hyperglycemia is associated with the cognitive decline seen in both types of diabetic patients [4]. Hyperglycemia alters multiple mechanisms, causing activation of protein kinase $\mathrm{C}$ activity and the polyol pathway, accumulation of sorbitol and advanced glycation end products, disturbances in $\mathrm{Ca}^{2+}$ homeostasis, and augmentation of reactive oxygen species (ROS) levels. Increased levels of ROS and free radical generation disturb oxidative metabolism balance, which leads to detrimental effects on cellular function [5]. The detrimental changes induced by ROS in the cell include depletion of endogenous antioxidants, energy insufficiency, mitochondrial dysfunction, protein and DNA damage, and inflammation.

Furthermore, oxidative stress causes activation of the pleiotropic transcription factor known as nuclear factor kappa B-light-chain-enhancer of activated $\mathrm{B}$ cells (NF-KB). NF-KB activation further increases the levels of proinflammatory mediators like proinflammatory cytokines [cyclooxygenase (COX)-2, inducible nitric oxide synthase (iNOS), tumor necrosis factor (TNF)- $\alpha$, and interleukin (IL)-6]. Several studies have reported the involvement of NF-KB activation in peripheral and central nervous system complications of diabetes [6,7].

Melatonin ( $\mathrm{N}$-acetyl-5-methoxytryptamine), a pineal hormone, is one of the well-studied pharmacological interventions that exert protective effects primarily by ROS scavenging activity. In addition, melatonin augments the antioxidant defense system and down-regulates inflammatory mediators [8]. Several studies have demonstrated the neuroprotective action of melatonin in neurodegenerative disorders, such as amyotrophic lateral sclerosis, Alzheimer's disease, Parkinson's disease, and multiple sclerosis $[9,10]$. In a previous study, melatonin was found to have a neuroprotective effect against streptozotocin (STZ)-induced neurobehavioral and neurochemical deficits [11]. The aim of the present study was to investigate the protective effect of melatonin against cognitive dysfunction induced by type 2 diabetes.

\section{EXPERIMENTAL}

\section{Animals}

Male Sprague-Dawley rats (120 - $140 \mathrm{~g})$ were used in this study. The animal study was approved by Institutional animal ethical committee of Tongji Medical College, China (ref. no. TMC/2015/03) and performed in accordance with the guidelines of the Association for the Assessment and Accreditation of Laboratory Animal Care International (AAALAC) for experimentation and animal use [12].

\section{Induction of diabetes}

All the animals were fed with HFD initially for fourteen days and thereafter single dose of STZ (35 mg/kg, i.p.) was administered to induce diabetes. HFD was provided to the animals throughout the study period. Rats with serum glucose level $\geq 250 \mathrm{mg} / \mathrm{dL}$ were considered diabetic animals and were further selected for the study. All the animals were divided into four groups $(n=12)$ such as control group animals received vehicle (p.o.); HFD group animals were fed HFD and then given single dose of STZ injection. After completion of 15 weeks, vehicle was administered (p.o.) for subsequent three weeks. HFD + STZ + M $10 \mathrm{mg} / \mathrm{kg}$ group of animals were fed HFD and then given single dose of STZ injection. After completion of 15 weeks, melatonin $(10 \mathrm{mg} / \mathrm{kg})$ was administered (p.o.) for subsequent three weeks. M $10 \mathrm{mg} / \mathrm{kg}$ group of animals were treated with melatonin (10 $\mathrm{mg} / \mathrm{kg}$ ) for three weeks after completion of 15 weeks. Object recognition task and Morris water maze test were performed on $15^{\text {th }}$ and $18^{\text {th }}$ week.

\section{Morris water maze (MWM) test}

Cognitive function in all the animals was assessed by MWM test. Circular tank with $60 \mathrm{~cm}$ of height and $180 \mathrm{~cm}$ of diameter was used in the study. A platform was placed in the tank $2 \mathrm{~cm}$ below the water level having the height $38 \mathrm{~cm}$ and $12.5 \mathrm{~cm}$ diameter which was visible to pups. Tank was divided into four different quadrants and different symbol was placed in the room to differentiate the different quadrants of tank. Training was given to all the pups for 4 days continuously. In each session 4 trials were given at an interval of $30 \mathrm{~s}$ in which animal was placed in any one of quadrant. The pups were kept as such for the duration of $20 \mathrm{sec}$ on the platform after it climbs on it. The time taken by the pups to reach the platform was considered as escape latency. However time spent in the target quadrant was estimated by removing the stage 
from the tank and pups were allowed to search the platform.

\section{Object recognition task (ORT)}

ORT was performed in a black colored wooden box $\left(50 \times 70 \times 50 \mathrm{~cm}^{3}\right)$. The animal was initially habituated to the ORT box for $10 \mathrm{~min}$ in a day for two consecutive days. On $3^{\text {rd }}$ day, the rat was placed for $5 \mathrm{~min}$ in the ORT box and then, two objects of dissimilar appearance (a blue colored truncated cone and a cube-shaped white block) were kept in two opposite corners of the ORT box. Thereafter, the animals were allowed to explore ORT box for another 5-min period and the time spent in exploring the objects was recorded. Thereafter, animals were returned to their respective cage. After of duration of 120 $\mathrm{min}$, the animals were placed in the ORT box for $5 \mathrm{~min}$, and the objects placed back in the ORT box, with the cube-shaped white block used in the previous session changed to a novel object (an ellipsoid-shaped red colored object). The time spent to explore objects was recorded for 5 min period. The animal was considered to be exploring when animal were facing, sniffing, or biting the object. ORT box and the objects were cleaned thoroughly with alcohol to avoid olfactory cues. Cognitive function was evaluated by estimating the time spent in the target quadrant, preference index.

\section{Assessment of glucose and insulin level in rat serum}

Blood was collected from the rat in Eppendorf tube and serum was separated out by centrifuging blood at $4{ }^{\circ} \mathrm{C}$ for the duration of 5 $\mathrm{min}$ at $2000 \times$. Level of glucose and insulin in the serum were determined by glucose oxidase method and ELISA kit (Millipore, USA) respectively.

\section{Preparation of brain tissue}

At the end of protocol all the animals were sacrificed by cervical dislocation and hippocampus was isolated from the separated brain. $50 \mathrm{mM}$ Tris- $\mathrm{Hcl}$ of $\mathrm{pH} 7.4$ and $300 \mathrm{mM}$ of sucrose was used to homogenate the brain tissue for $10 \mathrm{~min}$ at $10000 \mathrm{rpm}$. Supernatant was separated out for further study.

\section{Determination of ROS level}

ROS level was estimated by adding $50 \mu$ of tissue homogenate in $4.85 \mathrm{ml}$ of potassium phosphate buffer $(100 \mathrm{mmol} / \mathrm{L}$ and $\mathrm{pH}$ 7.4). The mixture was incubated for $15 \mathrm{~min}$ with 2', 7'dichlorofluorescindiacetate dye at $37^{\circ} \mathrm{C}$. After incubation, mixture was centrifuged at $12,500 \mathrm{~g}$ for $10 \mathrm{~min}$ at $4{ }^{\circ} \mathrm{C}$. The pellet obtained after centrifugation was mixed with $5 \mathrm{~mL}$ of $100 \mathrm{mmol} /$ LPBS on a vortex at $0{ }^{\circ} \mathrm{C}$ and subsequently incubated for $60 \mathrm{~min}$ at $37^{\circ} \mathrm{C}$. Fluorescence was measured at $488 \mathrm{~nm}$ for excitation and $525 \mathrm{~nm}$ for emission. ROS level was calculated as picomoles per min per $\mathrm{mg}$ of protein.

\section{Assessment of oxido-nitrosative stress}

Level of lipid peroxidation (LPO) was determined as per the previously reported method in the brain tissues. Amount of malondialdehyde (MDA) was estimated at $532 \mathrm{~nm}$. Hippocampal GSH content was estimating the absorbance at a wave length of $412 \mathrm{~nm}$ and the level of GSH was articulated as micromoles of GSH per milligram of protein. Nitrite level was determined using a commercially available Griess reagent (containing $0.1 \% \quad \mathrm{~N}$-(1-naphthyl) ethylene diamine dihydrochloride, $1 \%$ sulfanilamide and $2.5 \%$ phosphoric acid). Equal amount of sample and Griess reagent were mixed and then incubated in a dark place. After $15 \mathrm{~min}$, absorbance was measured at $540 \mathrm{~nm}$.

\section{Determination of proinflammatory cytokines and Brain-derived neurotrophic factor (BDNF)}

IL-6 and TNF- $\alpha$ level was determined by Rat ELISA kit purchased from Invitrogen Co, Carlsbad, CA, USA. Hippocampal BDNF level was estimated by BDNF Emax® ImmunoAssay System (Promega Corporation, USA). Furthermore, Hippocampus was homogenized and sonicated in 8 volumes of $0.1 \mathrm{M}$ ice-cold PBS buffer ( $\mathrm{pH}$ 7.4) containing protease inhibitor cocktail. The homogenate was centrifuged at $10,000 \mathrm{rpm}$ for $10 \mathrm{~min}$ at $4{ }^{\circ} \mathrm{C}$. The assay was carried out with the help of manufacturer's kit. The absorbance was measured at $450 \mathrm{~nm}$ in ELISA plate reader.

\section{Western blot assay}

Hippocampus was homogenized in $500 \mu \mathrm{L}$ of RIPA buffer containing protease and phosphatase inhibitor cocktail. Thereafter, homogenate was centrifuged at $12,000 \mathrm{~g}$ for 20 $\mathrm{min}$. The supernatant was collected and stored at $-80{ }^{\circ} \mathrm{C}$ for further analysis. The concentration of proteins in the sample was measured using previously described method. Equal amount of protein extracts were separated by SDSpolyacrylamide gel electrophoresis (SDS-PAGE) and then separated proteins transferred onto a nitrocellulose membrane. The membrane was blocked by $3 \%$ bovine serum albumin. Thereafter, membranes were incubated with 
respective primary antibodies for overnight at 4 ${ }^{\circ} \mathrm{C}$. Thereafter membranes were washed with TBST and then incubated with a horseradish peroxidase conjugated antibody for $2 \mathrm{~h}$ at room temperature. The antigen-antibody-peroxidase complexes were visualized using a colored reaction with BCIP-NBT. The quantitative analysis of bands was carried out with the help of Image J software.

\section{Statistical analysis}

Data are expressed as mean \pm SEM. Student's $t$ test was used to compare the difference between control and diabetic group. One-way analysis of variance (ANOVA) followed by post-hoc Tukey test was used to compare the various groups. Two-way repetitive measure ANOVA followed by post-hoc Bonferroni test was used to find the differences in MWM acquisition data. $P<0.05$ was considered statistically significant.

\section{RESULTS}

\section{Body weight, and serum glucose level and serum insulin levels}

After 15 weeks of diabetes induction, type 2 diabetic rats had significantly increased body weight $(p<0.01)$ and showed marked $(p<0.01)$ elevation of serum glucose and insulin levels compared with the control group (Table 1). Treatment with melatonin significantly reduced the serum levels of glucose and insulin in type 2 diabetic rats compared with levels in the diabetic control group.

\section{Type 2 diabetes-induced cognitive deficits}

Cognitive function was assessed using the Morris water maze (MWM) and an object recognition task (ORT) to investigate cognitive impairment in control and diabetic rats at the $15^{\text {th }}$ week of the study protocol. On the first day of the MWM trial, there were no significant differences between mean escape latencies of control and diabetic rats. However, two-way ANOVA revealed longer escape latencies on the second $(p<0.01)$, third $(p<0.001)$, and fourth $(p<0.01)$ days of the acquisition trial by the diabetic group compared with the control group. These results indicate learning deficits in diabetic rats (Figure 1A). Furthermore, a probe trial was conducted to assess memory retention in diabetic rats. It was found that diabetic rats spent significantly $(p<$ 0.01 ) less time in the target quadrant than control rats, indicating poor memory retention in diabetic rats (Figure $1 \mathrm{~B}$ ).

The effect of diabetes on recognition memory after the $15^{\text {th }}$ week of diabetes induction was assessed using ORT. One-way ANOVA revealed that the diabetic rats failed to discriminate between familiar and unfamiliar objects placed in the ORT box. Consequently, the preference index of diabetic group was significantly $(p<$ 0.01 ) higher than that in the control group (Figure 1C). Similar behavioral results were observed after the $18^{\text {th }}$ week of diabetic induction.

\section{Melatonin treatment ameliorated cognitive deficits}

The melatonin (10 $\mathrm{mg} / \mathrm{kg})$-treatment group displayed a significant reduction in escape latency on the second $(p<0.05)$, third $(p<0.01)$, and fourth $(p<0.05)$ days of the trial compared with the cisplatin-treated group (Figure 2A). Thus, melatonin treatment significantly reversed the type 2 diabetes-induced learning deficits. Furthermore, impairment in spatial learning observed in a probe trial was significantly reversed $(p<0.01)$ in diabetic animals after melatonin treatment (Figure 2B). An ORT method was used to verify the effect of melatonin treatment on memory in rats. The melatonin treatment group spent significantly $(p<0.01)$ more time exploring the novel object than diabetic rats, indicating improvement in memory function after melatonin treatment (Figure 2C). In addition, melatonin treatment alone in normal rats did not alter the cognitive function.

\section{Melatonin treatment mitigates dioxido- nitrosative stress}

Diabetic rats showed increases in oxidative stress as evidenced by a significant rise in levels

Table 1: Effect of melatonin on body weight, serum glucose level, and serum insulin level in a type 2 diabetic rat model

\begin{tabular}{lccc}
\hline Group & Body weight $(\mathbf{g})$ & $\begin{array}{c}\text { Serum glucose } \\
(\mathbf{m g} / \mathbf{d L})\end{array}$ & $\begin{array}{c}\text { Serum insulin } \\
(\mathbf{n g} / \mathbf{m L})\end{array}$ \\
\hline Control & $155 \pm 11.3$ & $102 \pm 4.7$ & $6.2 \pm 0.5$ \\
HFD+STZ & $242 \pm 15.6^{\# \#}$ & $249 \pm 17.8^{\# \#}$ & $9.5 \pm 0.65^{\# \#}$ \\
HFD+STZ+M 10 mg/kg & $231 \pm 13.8^{\# \#}$ & $151 \pm 9.3^{\star *}$ & $0.78 \pm 0.2^{*}$ \\
M $10 \mathrm{mg} / \mathrm{kg}$ & $143 \pm 9.2$ & $97.5 \pm 3.1^{\star *}$ & $0.61 \pm 0.11^{* *}$ \\
\hline Data are expressed as mean \pm SEM $(\mathrm{n}=6) .{ }^{\# \#} p<0.01$ compared to control group; ${ }^{*} p<0.01$ and ${ }^{* *} p<0.01$ \\
compared to diabetic control
\end{tabular}




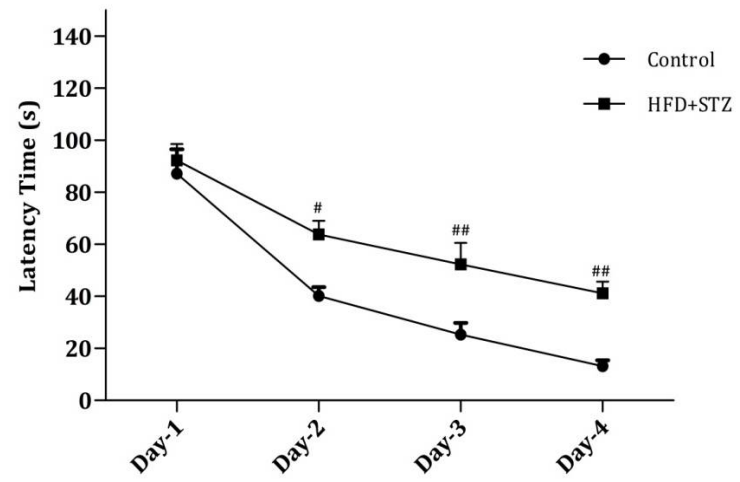

(a)

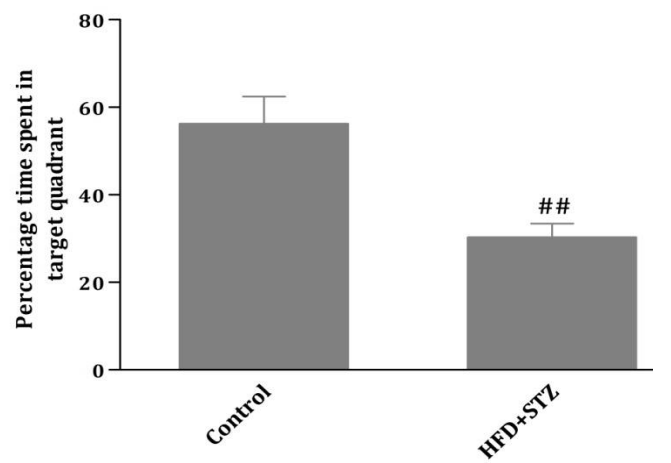

(b)

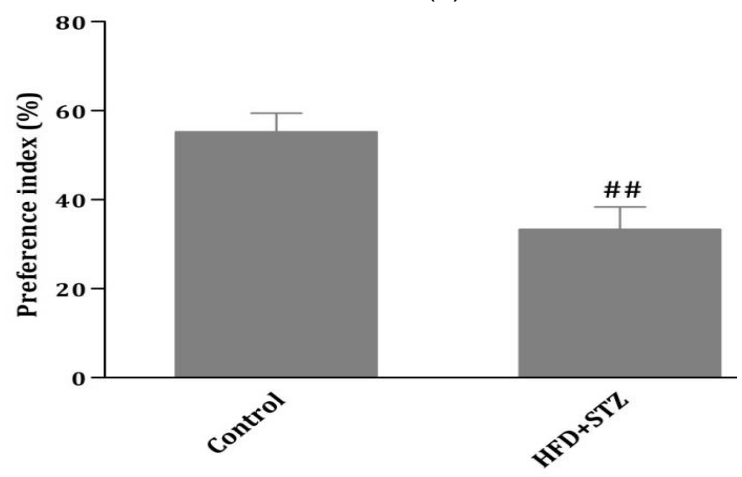

(c)

Figure 1: Effect of 15-week type 2 diabetes on (a) mean escape latency, (b) percentage time spent in the target quadrant, and (c) recognition memory. Data are expressed as mean \pm SEM $(\mathrm{n}=6)$. ${ }^{\#} p<0.05,{ }^{\# \#} p<0.01$ compared to control group

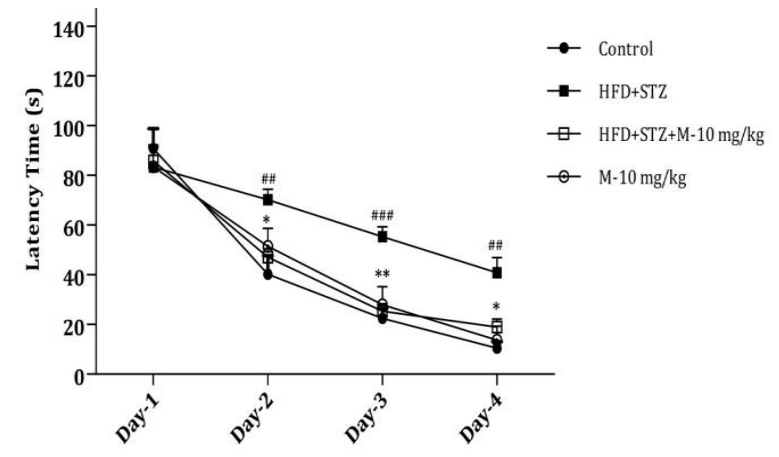

(a)

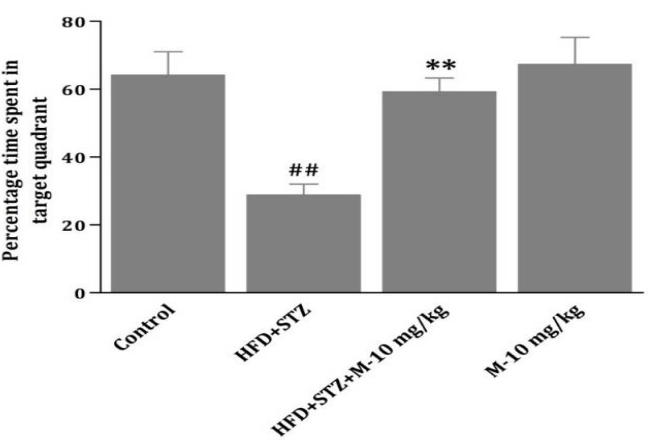

(b)

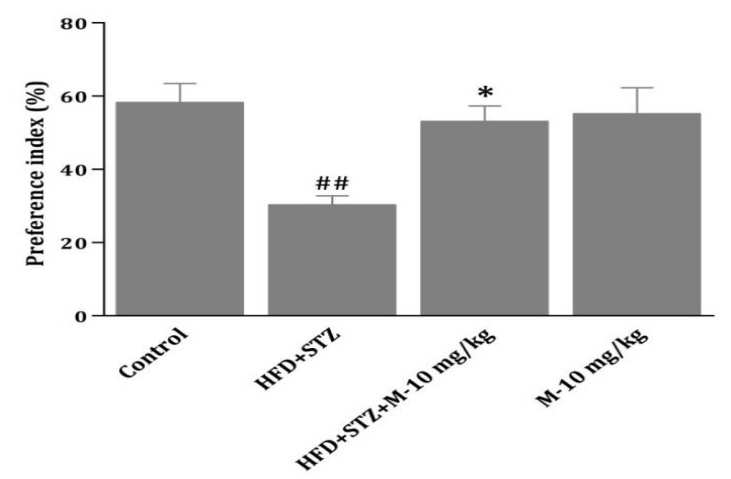

(c)

Figure 2: Effect of melatonin treatment on (a) mean escape latency, (b) percentage time spent in the target quadrant, and (c) recognition memory in diabetic rats. Data are expressed as mean \pm SEM $(n=6)$. ${ }_{* * *}^{\#} p<0.01$ and ${ }^{\# \# \#} p<0.001$ compared to control group; ${ }^{*} p<0.05,{ }^{* *} p<0.01$, and ${ }^{* * *} p<0.001$ compared to diabetic group 
of ROS $(p<0.01)$ and malondialdehyde (MDA; $p$ $<0.01$ ), and depletion of glutathione (GSH; $p<$ 0.01 ) in the hippocampus region (Figure 3 ). However, melatonin treatment was associated with significant reduction of oxidative stress by reducing MDA $(p<0.01)$ and $\operatorname{ROS}(p<0.01)$ levels in the hippocampus. Moreover, the GSH level was markedly $(p<0.01)$ increased in the hippocampus of melatonin-treated diabetic rats due to mitigation of oxidative stress by melatonin treatment. The nitrite level, an indirect indicator of nitrosative stress that was increased $(p<0.01)$ in the diabetic group, was significantly $(p<0.05)$ reduced by melatonin treatment (Figure 3D).

\section{Melatonin treatment neuroinflammation and prevented derived neurotrophic factor (BDNF) depletion}

Diabetic rats showed higher levels of hippocampal IL-6 and TNF- $\alpha(p<0.001)$ than normal animals (Figure 4). Treatment with melatonin caused a marked reduction in IL-6 $(p<$

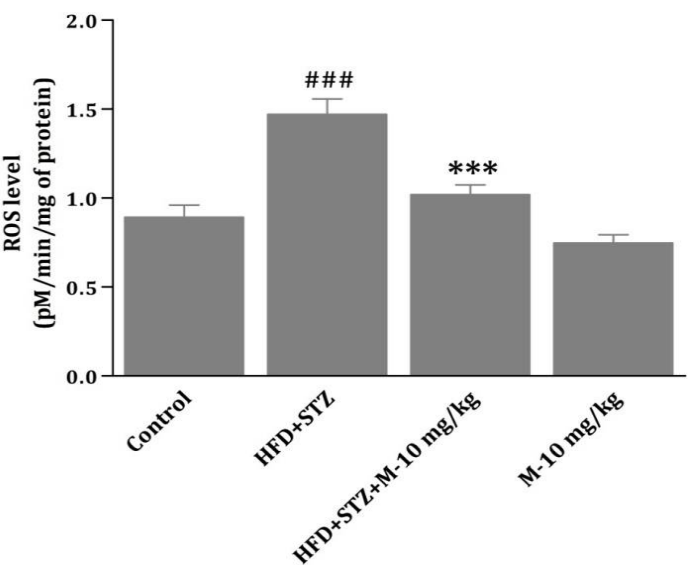

(a)

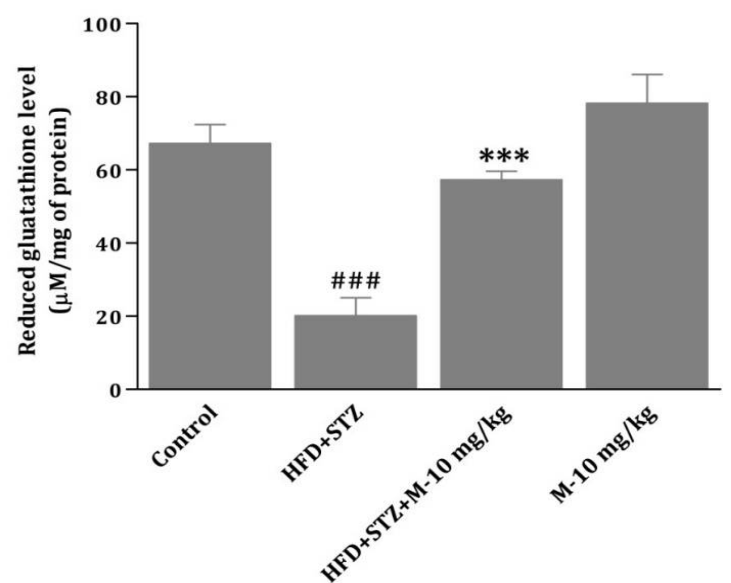

(c)
$0.001)$ and TNF- $\alpha(p<0.05)$ levels in diabetic animals. Thus, melatonin treatment significantly alleviated type 2 diabetes-induced neuroinflammation in the hippocampus region. Furthermore, hippocampal BDNF content was significantly $(p<0.001)$ depleted in the diabetic rats compared with control animals. On the other hand, melatonin treatment increased $(p<0.01)$ the BDNF level in the hippocampus compared with that in diabetic rats without melatonin treatment.

\section{Melatonin treatment inhibited NF-k $\beta$ activation}

The results in Figure 5 show that type 2 diabetes significantly $(p<0.001)$ increased the protein expression level of NF-KB and phosphorylated $\mathrm{IK} \beta$ in diabetic animals. Melatonin treatment significantly down regulated the NF-KB protein expression level $(p<0.001)$ and phosphorylation of $\operatorname{IK} \beta(p<0.05)$ in the diabetic rats.

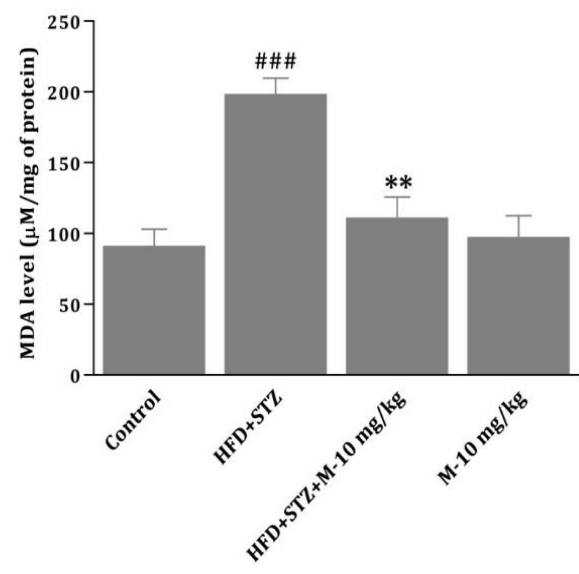

(b)

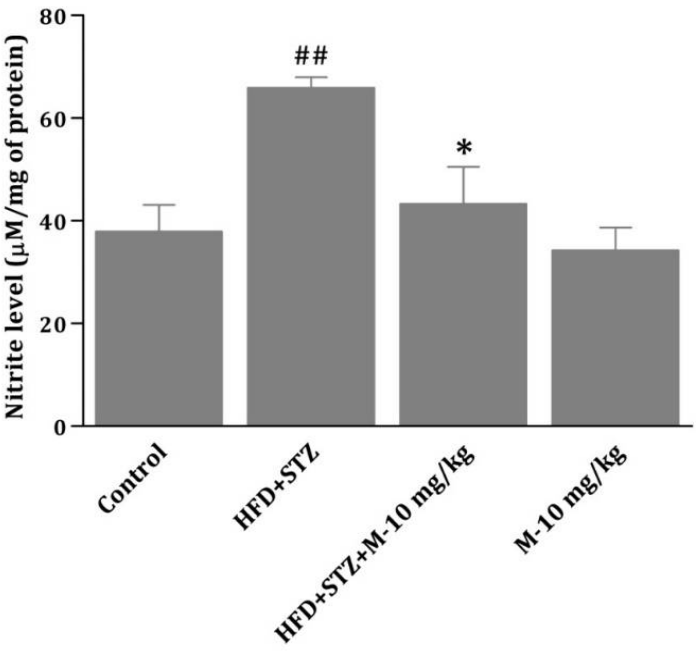

(d)

Figure 3: Effect of melatonin treatment on (a) ROS, (b) MDA, (c) GSH, and (d) nitrite level in the hippocampus of diabetic rats. Data are expressed as mean $\pm \operatorname{SEM}(n=6) .{ }^{\# \# \#} p<0.001$ compared to control group; $p<0.01$ and $p<0.001$ compared to diabetic group 


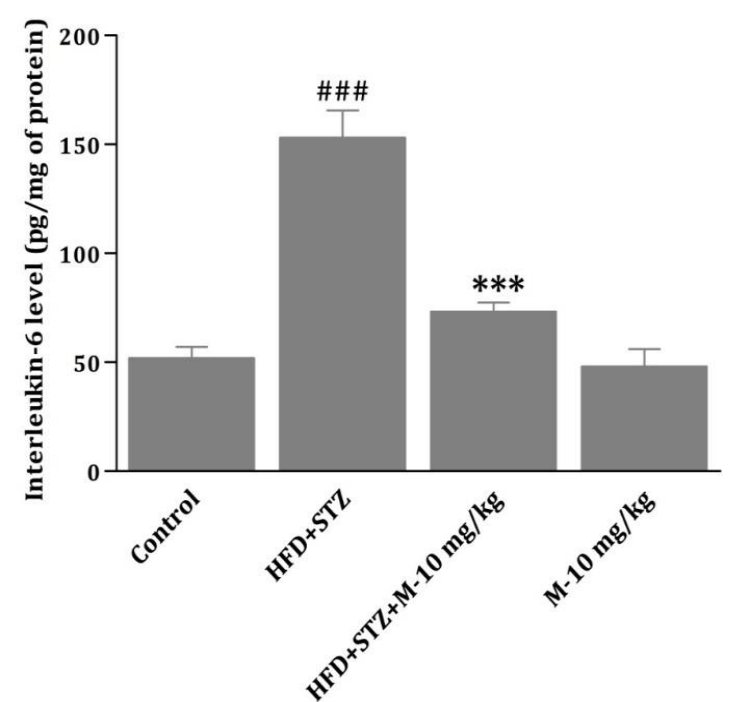

(a)

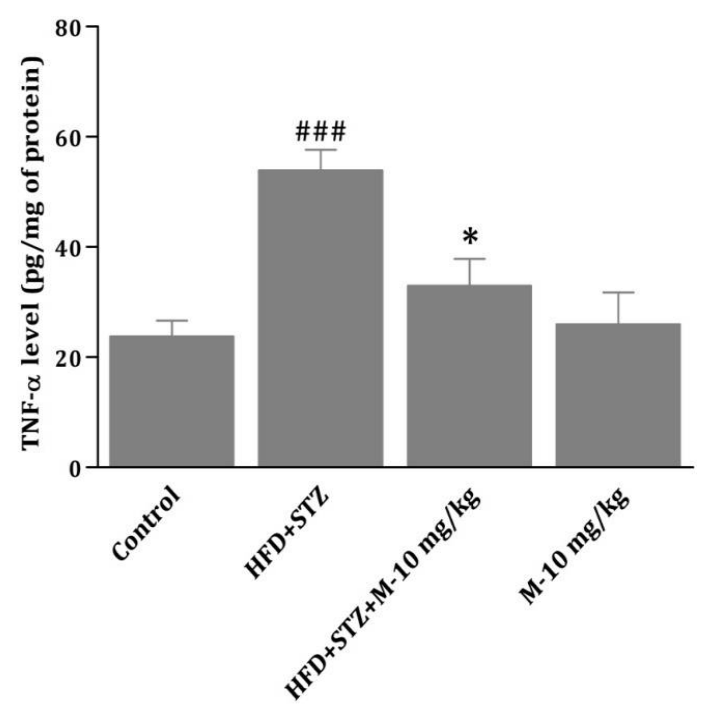

(b)

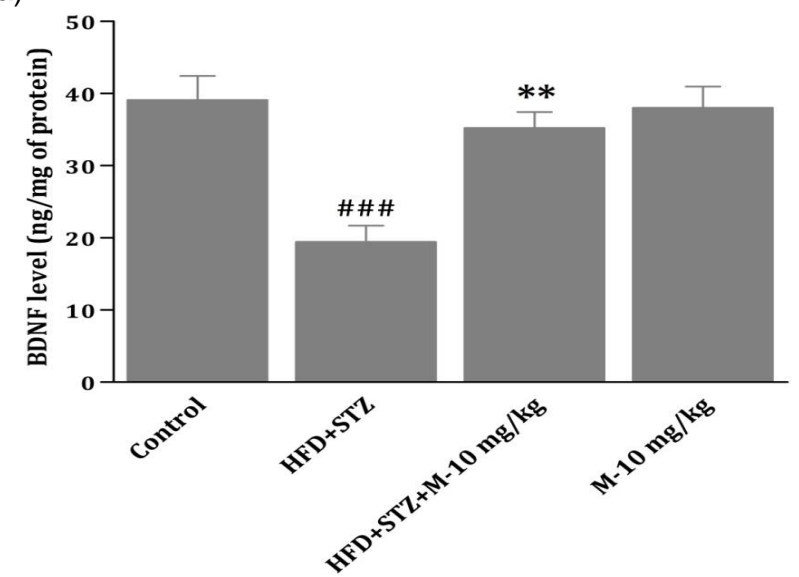

(c)

Figure 4: Effect of melatonin treatment on (a) IL-6, (b) TNF- $\alpha$, and (c) BDNF levels in the hippocampus of diabetic rats. Data are expressed as mean \pm SEM $(\mathrm{n}=6)$. \#\#\# $p<0.001$ compared to control group; $p<0.05,{ }^{* *} p$ $<0.01$, and ${ }^{* \star *} p<0.001$ compared to diabetic group

\section{DISCUSSION}

This study evaluated the protective effect of melatonin against cognitive dysfunction in type 2 diabetic rats. Previous experimental reports demonstrated that both type 1 and type 2 diabetes cause cognitive dysfunction via alterations in neurovascular and synaptic architecture [13]. In this study, an HFD with a single low-dose injection of STZ was used to induce type 2 diabetes in rats. Cognitive function was evaluated in diabetic animals on the $15^{\text {th }}$ week as evidenced by MWM and ORT results. The results regarding HFD + STZ-induced cognitive deficits are supported by previously reported studies [21]. Chronic treatment with melatonin for 21 days after 15 weeks of inducing diabetes significantly reversed the HFD + STZinduced cognitive deficits. The observed protective effect of melatonin against cognitive dysfunction is in accordance with previous experimental studies of melatonin [14]. On the $18^{\text {th }}$ week, HFD + STZ diabetic animals were characterized by hyperglycemia and exhibited significant learning and memory impairment.

This impairment was coupled with marked increases in oxido-nitrosative stress, neuroinflammation, NF-kB activation, and BDNF depletion in the hippocampus region. Treatment with melatonin for 21 days significantly alleviated the cognitive deficits by augmentation of BDNF levels and attenuation of oxido-nitrosative stress, neuroinflammation, and NF-kB in the hippocampus.

Hyperglycemia plays a crucial role in the pathogenesis of diabetes-induced cognitive dysfunction [15]. Hyperglycemia-induced ROS is considered a major culprit in the pathogenesis of diabetes-associated complications [16]. In the brain, ROS-generated oxidative stress disturbs the synaptic function and thereby causes cognitive impairment [17]. In the present study, 

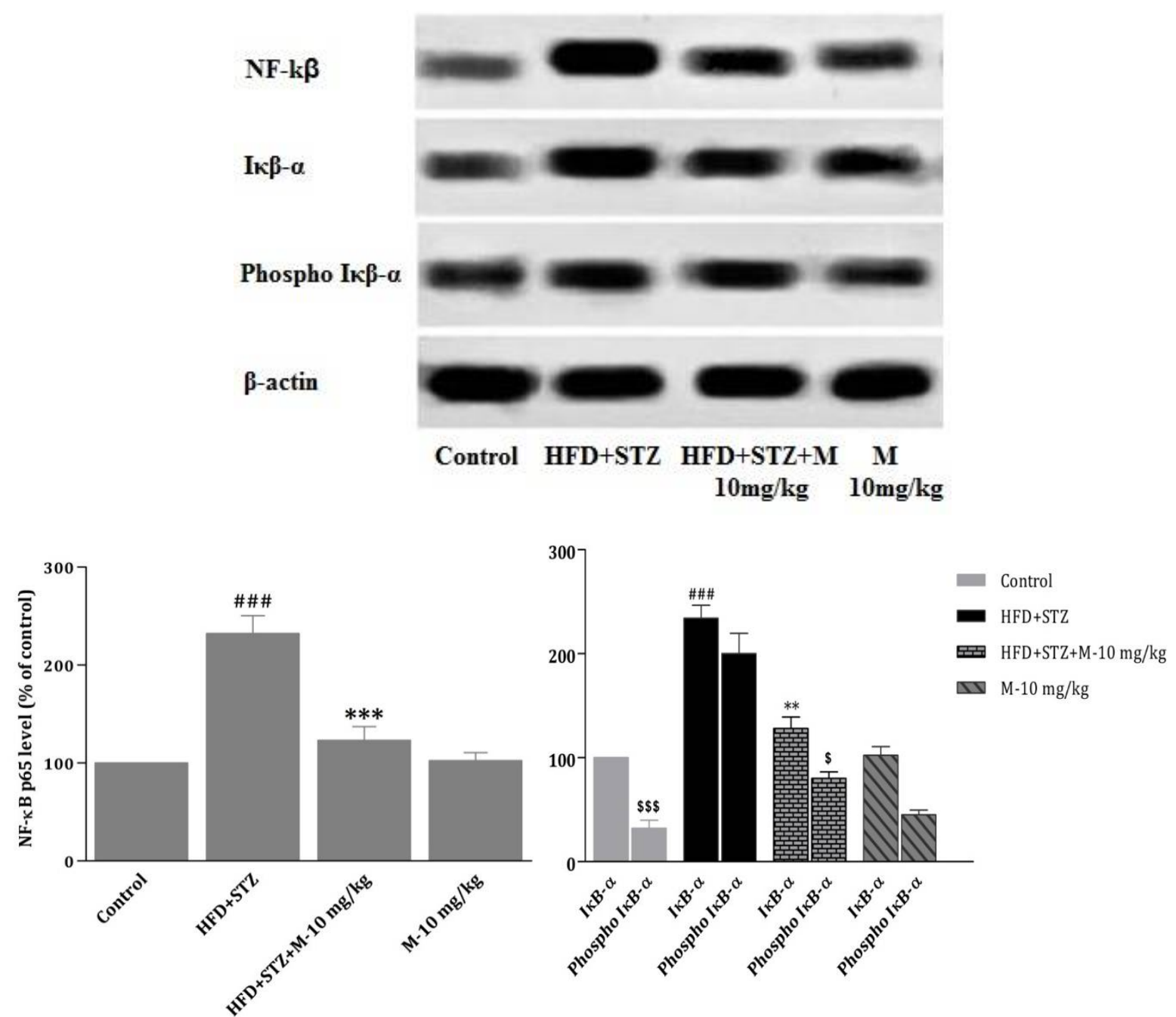

Figure 5: Effect of melatonin treatment on NF-KB, ІкB, and phospho-IKB expression levels in the hippocampus of diabetic rats. Results are expressed as mean \pm SEM $(n=3)$. \#\#\# $p<0.001$ compared to control group; $p<0.01$ and ${ }^{* * *} p<0.001$ compared to diabetic group; ${ }^{\$} p<0.05$ and ${ }^{\$ \$} p<0.001$ compared to IKB

the levels of MDA (a lipid peroxidation marker) and nitrite (an indicator of nitrosative stress) were elevated in the hippocampus region of the

diabetic group. Increased levels of oxidative stress deplete enzymatic and non-enzymatic antioxidants levels and disrupt the normal functioning of mitochondria, thereby exaggerating oxidative stress. The results showed that diabetes significantly depleted the GSH level in the hippocampus region. Melatonin and its metabolites are powerful free radical scavengers and augment the expression of several antioxidant enzymes [18-20]. In this study, melatonin treatment significantly alleviated oxidative stress as evidenced by reduction of MDA and nitrite level and augmentation of GSH level in the hippocampus.

The involvement of the NF-KB cascade in the pathogenesis of diabetes-induced complications is well documented in previous experimental studies [21]. The NF-KB signaling pathway has been implicated in a number of neurodegenerative diseases as well and it is activated by oxidative stress and proinflammatory cytokines [22]. Under physiological conditions, NF-KB remains in an inactive condition in the cytoplasm by forming a complex with the inhibitory kappa beta (IKß) family. However, in cases of activation, NF-kBs detach from $I \kappa \beta$ and translocate to the nucleus where they bind to the $k \beta$ sequence of DNA. This bond alters the gene expression level of several inflammatory mediator proteins, such as IL-6, TNF- $\alpha$, iNOS, and COX-2. These NF-kB induced inflammatory mediators eventually cause neuronal cell death and cognitive impairment [23]. Our results showed up-regulation of NF-k $\beta$ protein in the hippocampus of diabetic rats compared to normal rats. Proinflammatory cytokines (IL-6 and TNF- $\alpha$ ) were also significantly increased in the hippocampus of diabetic rats, which indicates profound 
neuroinflammation. Numerous studies suggest that the neuroprotective effect of melatonin is mediated by inhibition of the NF-KB cascade [24].

In the present study, similar findings were observed after chronic treatment with melatonin. Diabetes-induced NF-KB and its associated neuroinflammation were significantly reduced after 21 days of melatonin treatment. Moreover, the results revealed an increased level of nitrite in the diabetic animals, which indirectly indicates the up-regulation of iNOS level in the hippocampus. However, melatonin treatment caused marked reduction in the nitrite level of diabetic animals. The reduction of nitrite level by melatonin is attributed to its reactive nitrogen species (RNS) scavenging property and NF-kB inhibition activity.

\section{CONCLUSION}

The results of this study demonstrate that melatonin prevents cognitive dysfunction in type 2 diabetic rats by attenuating oxido-nitrosative stress and NF-K-mediated neuroinflammation. These results indicate that melatonin can potentially be used for the management of cognitive dysfunction in patients suffering from diabetes. However, further studies are required to confirm this.

\section{DECLARATIONS}

\section{Acknowledgement}

The authors thank the central hospital of wuhan, tongji medical college, Huazhong university of science and technology, China, for providing the facilities for this study.

\section{Conflict of interest}

The authors declare that no conflict of interest is associated with this study.

\section{Authors' contribution}

We declare that this work was done by the authors named in this article and all liabilities pertaining to claims relating to the content of this article will be borne by the authors. Zhang Xuyan performed the experiments and wrote the report. Ye Jing Ping, Wang Zhongjing, Ding Sheng, Li $\mathrm{Li}$, and Yang Fan analyzed data and gave comments. Mao Hong designed the project, supervised the experiments, and wrote the entire manuscript. All authors read and approved the final manuscript.

\section{REFERENCES}

1. Hu FB. Globalization of Diabetes: the role of diet, lifestyle, and genes. Diabetes Care 2011; 34: 12491257.

2. Tripathi AS, Mazumdar PM, Chandewar AV. Changes in the pharmacokinetic of sildenafil citrate in rats with Streptozotocin-induced diabetic nephropathy. J Diabetes Metab Disord 2014; 13: 1-6.

3. Tripathi AS, Mazumdar PM, Chandewar AV. Sildenafil, a phosphodiesterase type 5 inhibitor, attenuates diabetic nephropathy in STZ-induced diabetic rats. J Basic Clin Physiol Pharmacol 2016; 27: 57-62.

4. Kodl CT, Seaquist ER. Cognitive dysfunction and diabetes mellitus. Endocr Rev. 2008; 29: 494-511.

5. Smith MA, Zhu X, Tabaton M, Liu G, McKeel (Jr) DW, Cohen $M L$ et al., Increased iron and free radical generation in preclinical Alzheimer disease and mild cognitive impairment. J Alzheimers Dis. 2010; 19: 363372.

6. Kuhad A, Bishnoi M, Tiwari V, Chopra K. Suppression of NF-kappabeta signaling pathway by tocotrienol can prevent diabetes associated cognitive deficits. Pharmacol Biochem Behav 2009; 92: 251-259.

7. Kumar A, Sharma SS. NF-kappaB inhibitory action of resveratrol: a probable mechanism of neuroprotection in experimental diabetic neuropathy. Biochem Biophys Res Commun. 2010; 394: 360-365.

8. Dugo L, Serraino I, Fulia F, De Sarro A, Caputi AP, Cuzzocrea $S$. Effect of melatonin on cellular energy depletion mediated by peroxynitrite and poly (ADPribose) synthetase activation in an acute model of inflammation. J Pineal Res. 2001; 31: 76 - 84.

9. Rosales-Corral SA, Acuña-Castroviejo D, Coto-Montes A, Boga JA, Manchester LC, Fuentes-Broto L, Korkmaz A, Ma S, Tan DX, Reiter RJ. Alzheimer's disease: pathological mechanisms and the beneficial role of melatonin. J Pineal Res. 2012; 52: 167 - 202.

10. Agil A, Reiter RJ, Jiménez-Aranda A, Ibán-Arias $R$, Navarro-Alarcón M, Marchal JA, Adem A, Fernández VG. Melatonin ameliorates low-grade inflammation and oxidative stress in young Zucker diabetic fatty rats. $J$ Pineal Res. 2013; 54: 381 - 388.

11. Miller E, Morel A, Saso L, Saluk J. Melatonin redox activity. Its potential clinical applications in neurodegenerative disorders. Curr Top Med Chem. 2015; 15:163 - 169.

12. Guide for the Care and Use of Laboratory Animals: Eighth Edition Committee for the Update of the Guide for the Care and Use of Laboratory Animals; National Research Council. 2010; ISBN: 0-309-15401-4.

13. Jiang $L Y$, Tang SS, Wang $X Y$, Liu LP, Long $Y$, Hu $M$, Liao MX, Ding QL, Hu W, Li JC, Hong H. PPARY agonist pioglitazone reverses memory impairment and biochemical changes in a mouse model of type 2 diabetes mellitus. CNS Neurosci Ther. 2012; 18: 659666. 
14. Olcese JM, Cao C, Mori T, Mamcarz MB, Maxwell A, Runfeldt MJ, Wang L, Zhang C, Lin X, Zhang G, Arendash GW. Protection against cognitive deficits and markers of neurodegeneration by long-term oral administration of melatonin in a transgenic model of Alzheimer disease. J Pineal Res. 2009; 47: 82 - 96.

15. Biessels GJ, Koffeman A, Scheltens P. Diabetes and cognitive impairment. J Neurol. 2006; 253: 477 - 482.

16. Raza H, John A, Howarth FC. Increased oxidative stress and mitochondrial dysfunction in zucker diabetic rat liver and brain, Cell Physiol Biochem. 2015; 35: 1241 - 1251.

17. Soares $E$, Prediger $R D$, Nunes $S$, Castro $A A$, Viana $S D$, Lemos $C$, De Souza CM, Agostinho $P$, Cunha RA, Carvalho E, Fontes Ribeiro CA, Reis F, Pereira FC. Spatial memory impairments in a prediabetic rat model. Neuroscience 2013; 250: 565 - 577.

18. Tan DX, Chen LD, Poeggeler B, Manchester LC, Reiter RJ. Melatonin: a potent endogenous hydroxyl radical scavenger. Endocr J. 1993; 1:57-60.

19. Rodriguez C, Mayo JC, Sainz, RM, Antolín I, Herrera F, Martín V, Reiter RJ. Regulation of antioxidant enzymes: a significant role for melatonin. J Pineal Res 2004; 36: 1 -9 .

20. Hardeland R, Tan DX, Reiter RJ. Kynuramines, metabolites of melatonin and other indoles: the resurrection of an almost forgotten class of biogenic amines. J Pineal Res. 2009; 47: 109 - 126.

21. Patel S, Santani D. Role of NF-kappa B in the pathogenesis of diabetes and its associated complications. Pharmacol Rep. 2009; 61: 595 - 603.

22. Mincheva-Tasheva S, Soler RM. NF-KB signaling pathways: role in nervous system physiology and pathology. Neuroscientist. 2013; 19: 175 - 194.

23. Yirmiya R, Goshen I. Immune modulation of learning, memory, neural plasticity and neurogenesis. Brain Behav Immun 2011; 25: 181 - 213.

24. Beni SM, Kohen R, Reiter RJ, Tan DX, Shohami E, Melatonin-induced neuroprotection after closed head injury is associated with increased brain antioxidants and attenuated late-phase activation of NF-kappaB and AP-1.FASEB J. 2004; 18:149-51. 